\title{
The problem with registrar research
}

\author{
Annie E. A. Bartlett, Clinical Research Fellow in Forensic Psychiatry; and \\ LYNNE M. DRUMmOND, Consultant Psychiatrist and Senior Lecturer in \\ Behavioural Psychotherapy, St George's Hospital Medical School, London SW17 0RE
}

In recent years registrars on psychiatric training rotations have been encouraged to begin their research careers early. Most of them are in such (training posts) for three and a half years. A few with extensive previous experience in other areas of medicine will be in post for less than this. The usual pattern of work with such schemes is to change both posts and accompanying routine every six months. At no point during this time will they have time allocated for research per se unless they are a fortunate member of some of the training rotations which include this in the weekly timetable. Such time has to be begged, stolen or borrowed from the hapless consultant who is all too aware of the service shortfall in his or her own area. If the registrar is fortunate enough to find an accommodating senior, this may not be the situation in his or her next post, which renders long term research well nigh impossible.

A further difficulty for registrars who are ambitious enough to undertake their own project is to persuade overworked and underpaid ancillary staff of the merits of their project so as to be in a position to receive considerable and often necessary secretarial support. Without the privacy of their own office, a luxury afforded to few, research actually within their work setting is also an uphill struggle.

The importance of a registrar undertaking research, however, has been emphasised in several spheres. It is certainly considered essential for promotion to senior registrar grade by psychiatric trainees themselves (Junaid \& Staines, 1990). This belief is not entirely erroneous and, as many know, it is not unusual for a good senior registrar job to receive 40-50 applications, all of whom have the MRCPsych and appropriate training. In such a climate, it is not unreasonable that an Appointments Committee looks to evidence of extra academic or clinical worthiness before short-listing, let alone making an appointment; research is often a deciding factor.

The new Achieving a Balance (UK Health Departments, 1987) has added a further complication to the difficulty of junior doctors completing appropriate research projects. These proposals, a major hurdle for a junior doctor, will be between SHO and registrar level. Once accepted as a registrar, barring any major incidents, the junior doctor is guaranteed to progress to senior registrar and ultimately consultant level.
However, a restriction on the number of registrar posts available means that any creation of research registrar posts would decrease the number of clinical registrar posts. Thus, even if money is found for a research registrar in these days of underfunding, it is likely to be vetoed by the Regional Manpower Committee. Research registrar posts will presumably be replaced by the research SHO or clinical assistant post. However, it is doubtful whether junior doctors at SHO level will have the available expertise and knowledge to embark successfully on a research project without considerable senior input. It therefore appears that increasingly the sad state of affairs will be that doctors will be compelled to take up SHO research jobs to increase their attractiveness in the job market, and perform research projects in which they may have little interest, and be able to contribute little of their own experience in devising and completing such research.

\section{Problems}

\section{Time factor}

The impetus of this article comes from our recent involvement in a research project which, contrary to our original expectation, has spanned five years. In the course of conducting this research, important issues about the feasibility of conducting research as a psychiatric registrar in training came to light.

Our research consisted of a trial of two antidepressant drugs, trazodone and clomipramine, to investigate whether they provided any additional beneficial effects when combined with behavioural psychotherapy in the treatment of non-depressed subjects, with primary obsessive compulsive disorder. The study design was a randomised doubleplanned, double dummy, parallel group comparison. The treatment phase was ten weeks in duration with follow-up to two years. The project was begun in 1985 and it was anticipated that it was going to last one to two years. This time-scale was derived from our knowledge of the number of patients passing through the behavioural psychotherapy treatment unit. It did not seem over-ambitious to attempt to find 30 patients as part of a multi-centre study. Nevertheless, it took two and a half years to recruit 25 patients. Screening for the project was undertaken 
by the registrar researcher who experienced particular difficulty in persuading patients of the merits of taking drugs and indeed of the merit of being monitored as frequently as a rigorous protocol demanded. Screening for the project was rendered problematic by the registrar rotating round the region at six monthly intervals and by the difficulties of coordinating with the teaching hospital base given such disruption. Of necessity, a project which might better have been run on a daily basis by a succession of research registrars involved the same personnel throughout. Thus a project for which the research protocol was written in late 1984 was available only to be analysed in 1990. During the intervening period the multi-centre nature of the study had disintegrated such that the original number of patients needed was woefully inadequate. Therefore, despite the fact that the patients improved with behavioural psychotherapy for the purpose of statistical analysis, the data are unsatisfactory.

Following an exhausting process of the registrar squeezing patients' screening appointments into an already hectic work schedule at irregular intervals, travelling around the region to various screening sites, there is little to write up. Thus the opportunity for the registrar to enhance her training by writing up a thoroughly well organised drug study has been lost. The benefits that would come with such publication have largely been declined on the basis of a desire to maintain academic respectability. The time factor therefore means that even where a registrar is motivated to put effort into a properly conducted drug study, no major publications are likely to result. $\mathrm{Had}$ the registrar been somewhat less keen and engaged in a less time-consuming piece of work then a publication may have resulted which could have led to increased promotional prospects.

\section{Funding}

Our research received drug company funding. It is readily acknowledged by those involved in grant applications that this is by far the most likely way to achieve success in obtaining funding. While it is clearly desirable that biological psychiatry continues to test out new developments in the field, the prescriptive nature of the sponsorship has undesirable effects. It is clearly far easier for a junior researcher, who is unlikely to obtain funding from elsewhere, to opt for this brand of research than to attempt something in other fields of psychiatry. Isolated individuals, even those with well developed and original research ideas, who do not hail from prestigious centres, are unlikely to be more than glanced at by major charities. The situation is arguably worse in psychiatry than any other field of medicine as there are depressingly few charitable bodies who do fund psychiatric research e.g. the
Mental Health Foundation and the Medical Research Council have many more projects than they can hope to fund and tend to fund the large project with many research workers of known calibre.

Projects requiring equipment of any kind, e.g. computers, statistical packages, and which involve questionnaire and other postal costs, pose financial problems which are seldom overcome by the junior registrar. Some health authorities are more generous than others and some registrars work with consultant psychiatrists who are also senior lecturers and have some limited budgets themselves. However, for any registrar wanting to engage in a minor project, funding can prove a major difficulty.

\section{Comment}

Overall, it seems difficult for a registrar to complete a well conducted trial and to write up a research project on a part-time basis at the same time as sitting for the MRCPsych examination, which is also required for promotion. Although the position of research registrar is one answer to this, as has already been discussed, there is little chance of gaining the requisite funding, and due to the new Achieving a Balance procedures, these posts are likely to be decreasing rather than increasing in number. The registrar, therefore, who wants to gain a publication and continue in his or her career, has few options left. One possibility is to help out in an existing study and hope that the other co-workers will place his/her name somewhere on the finished publication. However, even this option is likely to be available only to those registrars working in a major teaching hospital where research is being undertaken on a regular basis. The other option is to aim for a lesser publication in a reputable journal. In St George's Hospital in the South West Thames Region in recent years there has been greater emphasis on the role of the single case study description which emphasises unusual or important clinical matters. In our region, encouragement has been given to registrars writing up single case studies by introducing a research prize which is awarded, on an annual basis, to the registrar who has written the best case history. Other suggestions of ways in which registrars may be expected to undertake research have been made elsewhere (Crisp, 1990).

\section{References}

CRISP, A. H. The case for teaching and research experience and education within basic specialist training (registrar grade) in psychiatry. Psychiatric Bulletin, 14, 163-168.

JUNAID, O. \& Staines, J. (1990) Career progression in psychiatry: perceptions and realities. Psychiatric Bulletin, 14, $484-486$.

UK Health Departments, the Joint Consultants Committee and Chairmen of Regional Health AUTHORITIEs (1987) Hospital Medical Staffing: Achieving a Balance. Plan for Action. London: HMSO. 